\title{
Neuartige Wege des Handelns und Denkens
}

Innovationen sind für die Anpassung der ländlichen Wirtschaft an veränderte Rahmenbedingungen von besonderer Bedeutung. Die eigentlichen Innovationsprozesse entstehen dabei durch Kooperationen innerhalb von regionalen Netzwerken. Doch wie können solche Netzwerke zukünftig besser unterstützt werden? Von Karlheinz Knickel und Sarah Peter

nnovationen, seien sie organisatorischer, sozialer oder technischer Art, stellen hinsichtlich Herausforderungen wie Klimawandel und Energiekrise ein Kernelement von Problemlösungsstrategien dar. Gleichzeitig können Innovationen die Wettbewerbsfähigkeit steigern und zu nachhaltigen Produktions- und Wirtschaftsformen beitragen.

Die Innovationsprozesse für die nachhaltige Entwicklung ländlicher Gebiete wurden im Rahmen des EU-Projekts In-Sight von sieben interdisziplinären Forschungsteams untersucht. Dabei wurden beispielhaft drei Schwerpunktbereiche analysiert: Nutzung von Biomasse als regenerative Energiequelle unter besonderer Berücksichtigung von Reststoffverwertung und Energiepflanzenanbau, die Etablierung neuer Vermarktungswege für landwirtschaftliche Erzeugnisse und die Einführung neuer Dienstleistungen im ländlichen Raum. Am Bereich Bioenergie lässt sich das vorherrschende Verständnis von Innovationsprozessen besonders gut kritisch beleuchten.

\section{Die Rolle von Innovationen}

Integrierte, multifunktionale und post-produktivistische Perspektiven einer nachhaltigen Entwicklung ländlicher Räume müssen konsequenterweise zu einer Infragestellung unseres bisherigen sektoral geprägten Innovationsverständnisses führen. Hierauf verweisen unter anderem van der Ploeg et al. (2000), Knickel (2005) sowie Wilson (2007). Die reformierte Gemeinsame Agrarpolitik und die neuere EU-Politik zur ländlichen Entwicklung unterstützen diese Sicht (CEC 2006). Die Neuorientierung umfasst neben einer Umstrukturierung der ländlichen Wirtschaft auch Umweltbelange und die Frage der Lebensqualität in ländlichen Räumen. Dies sind Aspekte, die zunehmend auch als Plattform und Ausgangspunkt für wirtschaftliche Diversifizierung und Nachhaltigkeit wahrgenommen werden.
Vor diesem Hintergrund bietet die Erschließung neuer Märkte und neuer Dienstleistungen in vielen Regionen die Möglichkeit einer Verbreiterung der Einkommensbasis und der Schaffung neuer Arbeitsplätze. Beispielhaft sind die Installation von Windkraft-, Solar- und Biogasanlagen sowie der Anbau von Energiepflanzen. Im Idealfall sind diese mit dem Aufbau regionaler Wertschöpfungsketten für erneuerbare Energien und dezentraler Energiesysteme verbunden.

Kennzeichnend für Innovationsprozesse ist in vielen Bereichen, dass technologische Veränderungen mit organisatorischen und sozialen Veränderungen einhergehen. Für den Erfolg von Innovationsprozessen ist es daher ausschlaggebend, dass das Zusammenwirken von Neuerungen in unterschiedlichen Bereichen berücksichtigt wird, was vor allem in technologiefokussierten Prozessen oft nicht der Fall ist.

So sind Entwicklungen im Bereich Bioenergie oft nur dann nachhaltig, wenn der Einsatz neuer Technologien mit der Etablierung von regionalen Wertschöpfungsketten und dezentralen Energiesystemen gekoppelt wird. Zusätzlich zur technologischen Dimension ist dann die Frage neuer Kooperationen relevanter regionaler Akteure zu betrachten, wobei der Aufbau von Partnerschaften besonders in der Anfangsphase eines Innovationsprozesses von besonderer Bedeutung ist. Gerade im Bereich der erneuerbaren Energien entstehen oftmals komplexe regionale Netzwerke, in die ein breites Akteursspektrum involviert ist. Diese Prozesse erfordern unter anderem ein professionelles und langfristiges Management der Kooperationsnetzwerke.

\section{Unterschiedliche Wissensformen}

In der hier eingenommenen Perspektive reichen technische und ökonomische Einflussfaktoren allein nicht aus, um den Erfolg oder das Scheitern von Innovationsprozessen zu erklären. Letztere sollten vielmehr immer als Ergebnis der Kooperation innerhalb von und zwischen Netzwerken verstanden werden, in denen Informationsaustausch und Lernprozesse stattfinden.

Neuerungen bezeichnen, der Definition van der Ploegs (2003) folgend, die Erschließung „neuartiger Wege des Handelns und Denkens, einen neuen Modus, der Verbesserungspotenzial gegenüber etablierten Praktiken birgt“. Die Kapazitäten der ländlichen Akteure und Wirtschaft, neue Erkenntnisse, Praktiken, Produkte und neue Kombinationen von Ressourcen und technischen Verfahren zu realisieren sowie unterschiedliche Wissensbestände zusammenzuführen, sind somit von besonderer Bedeutung. 
Den Unterschied zwischen Innovationen und Neuerungen machen Wiskerke und van der Ploeg (2004) an der Art des mobilisierten Wissens fest. Anders als Innovationen basieren Neuerungen gemäß ihrem explorativen Charakter zumindest in der Anfangsphase auf sogenanntem nicht formalisierten Wissen oder „tacit knowledge“, das auch als lokales Wissen bezeichnet wird und mit dem Konzept der „art de la localité“ aus der ländlichen Soziologie vergleichbar ist. Dieser Wissensform wird als kontext- oder lokal- beziehungsweise regionalspezifischer Ressource von Oostindie, Broekhuizen (2008) hohe Relevanz für die ländliche Entwicklung beigemessen. Die besondere Bedeutung der Territorialität, die sich in der vornehmlichen Nutzung lokaler und regionaler Ressourcen sowie entsprechenden Netzwerken und Zielen ausdrückt, wird auch durch die empirische Forschung im Rahmen von In-Sight als Erfolgsfaktor bestätigt. Als Ergebnis lokalspezifischer Wissensbestände sind Neuerungen naturgemäß immer auch stärker ortsgebunden in ihren Einsatzmöglichkeiten, während Innovationen als Ausdruck kodifizierten Wissens mehr oder weniger global übertragbar sind (Oostindie, Broekhuizen 2008) (1). Gleichwohl kann aus Neuerungen kodifiziertes Wissen abgeleitet werden (Wiskerke 2004).

Die Schaffung von Neuerungen findet typischerweise in Netzwerken statt, die nicht von den in den vorherrschenden Akteurs- und Ressourcenkonstellationen gültigen Ansätzen durchdrungen sind. Ein charakteristisches Beispiel hierfür sind Basisinitiativen zur ländlichen Entwicklung, die sich bewusst für pflanzenölbasierte Technologien entschieden und diese maßgeblich weiterentwickelt haben. Diese Netzwerke stellen einen Raum dar, in dem Normen, Vorschriften, Produktionsverfahren, Distributions- und Konsummuster et cetera weniger strikt geregelt sind und, wohl auch deshalb, einer stärkeren Entwicklungsdynamik unterliegen. van der Ploeg et al. (2008) geht davon aus, dass in ländlichen Räumen das Verhältnis von kodifiziertem zu nicht kodifiziertem Wissen weit ausgeglichener ist als in urbanen Räumen. Das hiermit verbundene Potenzial kann als eine günstige Voraussetzung für die Aufnahme neuartiger Aktivitäten und den Aufbau neuer Netzwerke als Antwort auf veränderte gesellschaftliche Herausforderungen gesehen werden.

\section{Nicht-Linearität von Innovationsprozessen}

Abbildung 1 veranschaulicht das im Rahmen von In-Sight entwickelte nicht-lineare Modell der Strukturierung von Innovationsprozessen, unter Bezugnahme auf den Bioenergie-Bereich. Unterschieden werden hierbei vier Stufen: Erstens „novelties“, das Durchbrechen gewohnter Praktiken durch individuelles oder kollektives Handeln, zweitens „niches“, drittens „regimes“ und viertens „landscapes“, der zunehmenden Bedeutung neuartiger Strukturen auf höherer Ebene. Diese sind jedoch weder als voneinander isolierte Stadien zu betrachten, noch müssen sie notwendigerweise aufeinanderfolgen oder im Zuge eines Innovationsprozesses vollständig durchlaufen werden. Typisch für den Bereich Bioenergie, und hier vor allem Bio- kraftstoffe wie Biodiesel, ist der hohe Grad der vertikalen Integration von Rohstofferzeugung, Verarbeitung und Vermarktung sowie die teils rasche Maßstabsvergrößerung. Viele Energiepflanzenverarbeiter und Betreiber industrieller Anlagen steigen in einem relativ frühen Stadium des Betriebsaufbaus in globale Versorgungsketten ein, mit teils problematischen sozioökonomischen und ökologischen Konsequenzen.

In der Praxis folgt das Innovationsverständnis oft noch immer einem linearen Modell, wobei Innovation scheinbar als Ergebnis eines Flusses neuen Wissens stattfindet. Diesem Verständnis zufolge findet das in der Grundlagen- und dann vor allem in der Anwendungsforschung generierte Wissen erst im nächsten Schritt seinen Weg zum Anwender, zum Beispiel im Rahmen eines Produktionsprozesses. Im Falle der Wirtschaftlichkeit wird die Innovation dann von anderen Unternehmen übernommen oder durch Innovationstransferstellen und, im Agrarbereich, durch Beratungsdienste verbreitet (Godin 2005).

Die neuere, interdisziplinär ausgerichtete Innovationsforschung hat sich dagegen zunehmend dem Aspekt der kontextspezifischen Lernprozesse von Individuen und Organisationen zugewandt (Brunori et al. 2008). Dabei wird immer stärker eine systemische Perspektive eingenommen (Hubert et al. 2005, Knickel 2008). Im Projekt In-Sight wurde diese Sichtweise anhand von 14 empirischen Vertiefungsstudien bestärkt (Knickel et al. 2009).

In den Vertiefungsstudien wird deutlich, dass Innovationen fast zwangsläufig zu strukturellen Veränderungen im Verhältnis zwischen sozialen Akteuren und technischen Ressourcen und Verfahren sowie natürlichen Ressourcen führen. In diesem Verständnis erfolgt Innovationstransfer als Übertragung von einer Akteurs-Ressourcen-Konfiguration auf eine andere, wobei es sich um einen komplexen Anpassungsvorgang handelt, der Lernprozesse voraussetzt (Brunori et al. 2008; Knickel 2009).

Abbildung 1: Rahmenbedingungen und Dynamik der Innovationsentwicklung im Bereich Bioenergie

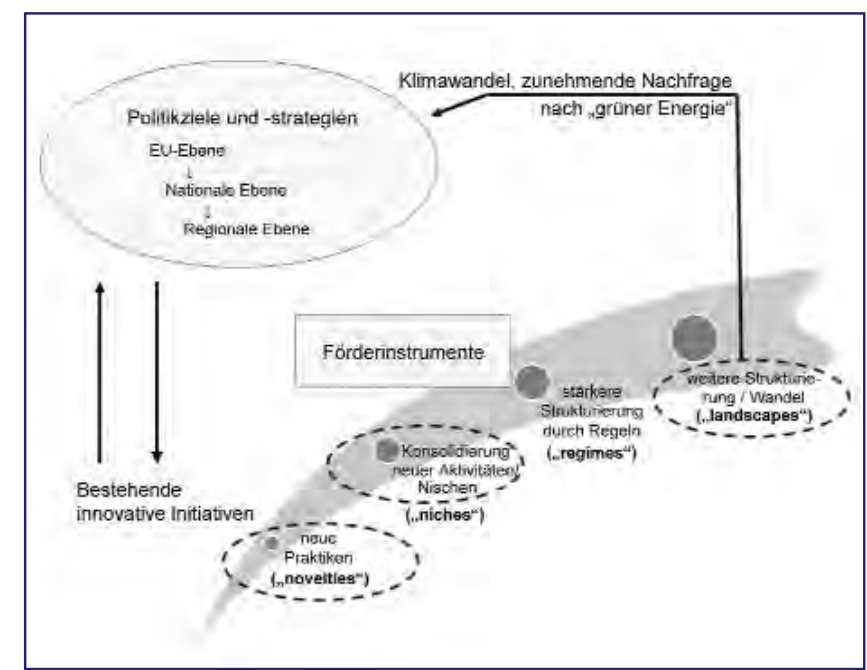

Quelle: Eigene Darstellung nach Rand et al. 2008 


\section{Zukünftige Innovationsförderung}

In der jüngeren Wissenschaftsdebatte wird eine zunehmende Divergenz zwischen sogenannten landwirtschaftlichen Wissens- und Informationssystemen oder Agricultural Knowledge and Information Systems (AKIS) und den aktuellen gesellschaftlichen und globalen Herausforderungen und Anforderungen thematisiert (van der Ploeg et al. 2008; Brunori et al. 2008; IAASTD 2008). Schon 2003 konstatierte van der Ploeg, dass landwirtschaftliches Wissen in der Form, wie es durch Expertensysteme, also Anwendungs- und universitäre Forschung, Ressortforschung der Ministerien und agroindustrielle Forschung, generiert wird, die realen Anforderungen vieler Akteure im ländlichen Raum und oft auch die öffentlichen Interessen verfehlt.

In einer zunehmenden Zahl von Analysen wird die wachsende Spezialisierung und Segmentierung der Bestände und Bereiche des Wissenssystems problematisiert (Hubert et al. 2005, IAASTD 2008). Gleichzeitig wird Wissen und Wissensmanagement zunehmend als logische Fortsetzung der Überlegungen zu lernenden Organisationen gesehen. Als Reaktion auf die zunehmende Kritik an ihrer Ineffizienz, hohen Bürokratisierung und Praxisferne wird das sogenannte Wissensdreieck aus Forschung, Beratung und Bildung, und hier vor allem die staatlich finanzierten Bereiche des AKIS, seit einiger Zeit einer starken Umstrukturierung unterzogen. Dies führte zu einer Privatisierung und Ausdehnung des Beratungswesens sowie zunehmendem Wettbewerb im Bereich der Forschung und Beratung (Kidd et al. 2000). Gleichzeitig haben privatwirtschaftliche Ziele in der öffentlichen Förderung an Bedeutung verloren und es ist eine Schwerpunktverlagerung hin zu sektorübergreifenden und regionsbasierten Maßnahmen erkennbar. Ob hiermit langfristig eine bessere Ausgewogenheit zwischen ökonomischen, ökologischen und sozialen Zielen erreicht werden kann, ist abzuwarten.

Abbildung 2: Förderliche Rahmenbedingungen der Innovationsentwicklung im Bereich Bioenergie

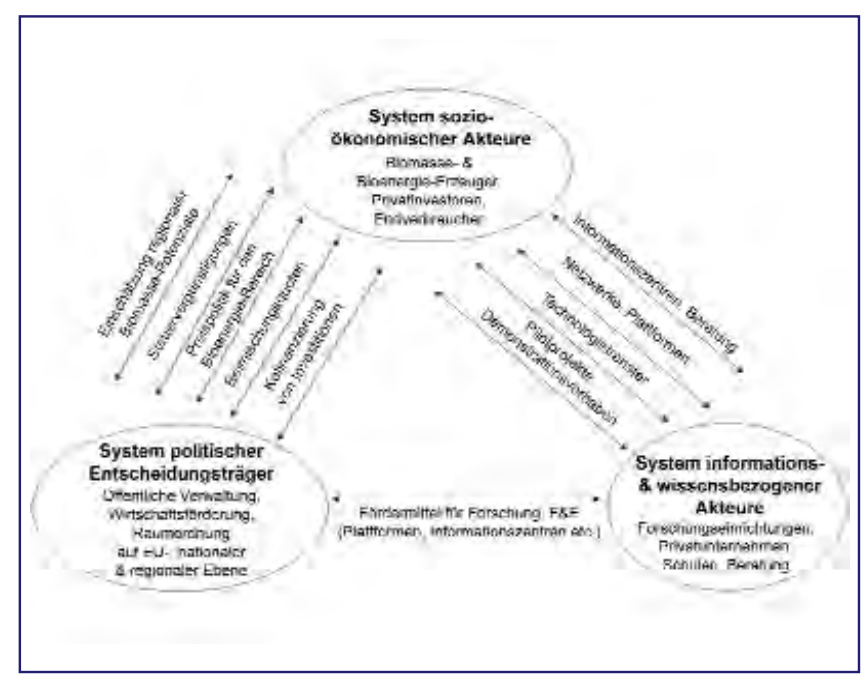

Quelle: Rand et al. 2008
Indessen richtet sich der politische Fokus zunehmend auf die Erkenntnis, dass Innovationen im produktiven Bereich nicht automatisch positive Auswirkungen auf ländliche Räume haben, sondern dass vielmehr oft ein Konfliktpotenzial hinsichtlich nachfrageorientierter Innovation einerseits und öffentlichen Interessen und Nachhaltigkeitsanforderungen andererseits besteht. Vor allem im Zuge der Diskussionen um Kosten und Nutzen der Gentechnik in der Nahrungsmittelproduktion sind öffentliche Interessen stärker ins Blickfeld gerückt.

Gerade im Zusammenhang mit öffentlichen Interessen und Nachhaltigkeitsanforderungen sind Innovationen über den technischen Aspekt hinaus zu betrachten. Oft muss es vor allem darum gehen, neue Strategien und Organisationsformen zu entwickeln, die technologische Neuerungen in ihren positiven Wirkungen effektiv unterstützen. Die erforderlichen Anpassungen beschränken sich hierbei zumeist nicht auf Einzelunternehmen, sondern sie betreffen eine Vielzahl an Akteuren gleichzeitig und können zu einer Neuordnung sozialer Beziehungsmuster führen.

\section{Lernprozesse und soziale Interaktion}

Diesem Verständnis entsprechend ist Innovation eng verknüpft mit Informationsflüssen, Lernprozessen und sozialer Interaktion. Als einer der Ersten führte Bandura (1977) das Konzept des sozialen Lernens ein, um zu erklären, wie soziale Beziehungsstrukturen und individuelle Lernprozesse einander wechselseitig beeinflussen. Eine wachsende Zahl von Studien und Projekten unterstreicht diesen Aspekt und hat demonstriert, wie soziales Lernen einen Beitrag zur Erreichung von Umwelt- und ländlichen Entwicklungszielen leisten kann (2).

Innerhalb sozialer Lernprozesse spielen unterschiedliche Wissensformen eine wichtige Rolle. Nicht formalisiertes Wissen wird generiert durch direkte Erfahrung beziehungsweise Learning by Doing, sodass sein Transfer persönliche Interaktion erfordert, während die Weitergabe kodifizierten Wissens mittelbar erfolgen kann (3). Innovationsprozesse weisen deshalb, wenn auch weniger ausgeprägt als Neuerungen, eine Tendenz zur geografischen Konzentration auf, weil nicht alle kontextspezifischen Komponenten von der Stufe des nicht formalisierten Wissens weiter übertragbar sind.

In den Projektergebnissen wird die Empfehlung einer Kooperation zwischen formellen und informellen Wissensnetzwerken formuliert, wobei Erstere als sehr relevant betrachtet werden. So spielen etwa in der deutschen Bioenergie-Fallstudie regionale Universitäten und Forschungsinstitute eine entscheidende Rolle für die Einführung neuer Technologien zum Holzeinsatz in der kommunalen Wärmeversorgung. In der Fallstudie war es das durch örtliche Behörden unterstützte koordinierte Zusammenspiel des Akteursspektrums, das auch Landwirte, Waldbesitzer, Energieversorger und Verbraucher umfasst, welches zum Erfolg führte.

Im Hinblick auf die Innovationsförderung werden im Rahmen des Projektes In-Sight drei Schlüsselakteursgruppen $\rightarrow$ 
identifiziert, die, wie in Abbildung 2 für den Bereich der Bioenergie dargestellt, sozioökonomische Akteure, politische Entscheidungsträger sowie informations- und wissensbezogene Akteure umfassen. Ausschlaggebend für eine nachhaltige Entwicklung im Bereich Bioenergie und allgemein für die Nutzbarmachung von Innovationen in der Entwicklung nachhaltiger Wirtschaftssysteme dürfte sein, wie gut diese Schlüsselakteursgruppen in Kooperationen, die der jeweiligen Problemstellung Rechnung tragen, zusammenwirken. Nicht vernachlässigt werden sollten hierbei dezentral organisierte Akteurs-Ressourcen-Konfigurationen sowie regional und lokal angepasste Technologien.

\section{Fazit}

Innovationen sind für die Anpassung der ländlichen Wirtschaft an veränderte gesellschaftliche Rahmenbedingungen und Nachhaltigkeitsanforderungen von besonderer Bedeutung. Die eigentlichen Innovationsprozesse sind hierbei als Ergebnis der Kooperation innerhalb von und zwischen Netzwerken zu verstehen, in denen Informationsaustausch und Lernprozesse stattfinden. Wichtige Neuerungen, etwa im Bereich der Bioenergie, werden begünstigt durch einen ausreichenden Entwicklungsspielraum in der Anfangsphase. In Hinblick auf die zukünftige Ausgestaltung der Innovationsförderung stellt sich die Schlüsselfrage, wie die formellen Wissensnetzwerke, Sektorforschung, Beratung und Bildung, dazu motiviert werden können, noch stärker und gezielter als bisher auf die eingangs beschriebenen Herausforderungen zu reagieren und vielversprechende Praxisentwicklungen auf regionaler und lokaler Ebene aufzugreifen und zu unterstützen.

\section{Anmerkungen}

(1) Kodifiziertes Wissen oder explizites beziehungsweise externalisiertes Wissen existiert in gespeicherter Form und verbirgt sich in vielfältiger Weise in Datenbanken, Schriften, Plänen, Karten usw.

(2) Beispiele sind die EU-Projekte LEARN:

http://www.inra.fr/learning/index.htm, HarmoniCOP: http://www.usf. uni-osnabrueck.de/ pahl/projekte/harmonicop/index.en.html, SLIM: http://www.slim.open.ac.uk und ETUDE: http://www.etuderd.eu/.

(3) Siehe hierzu Kenneth Arrow und seine Theorie des endogenen Wachstums im Zusammenhang von Innovation und technologischem Wandel (Arrow 1962).

\section{Literatur}

Arrow, K. J.: The economic implications of learning by doing. Review of Economic Studies 29/1962. S.155-73.

Bandura, A.: Social learning theory. Englewood Cliffs, NJ 1977.

Brunori, G. / Rand, S. / Proost, J.: Towards a conceptual framework for agricultural and rural innovation policies. IN-SIGHT-Project. Frankfurt am Main 2008.

CEC: Factsheet: The EU rural development policy 2007-2013. Internet: http://ec.europa.eu/agriculture/ publi/fact/rurdev2007/en_2007.pdf. 2006

Godin, B.: The linear model of innovation: Historical construction of an analytical framework. Project on history and sociology of S\&T statistics. Working paper No. 30. Internet: http://www.csiic.ca/PDF/Godin_30.pdf. 2005
Hubert, B. / Avelange, I. / Proost, J. / Ison, R. / Blackmore, C.: LEARNing in European agricultural and rural networks: Institutions, networks and governance. Final report. Contract no. HPSE-CT-2002-60059. 2005.

IAASTD: International assessment of agricultural science and technology for development. Washington / Rome 2008.

Kidd, A. / Lamers, J. / Ficarelli, P. / Hoffmann, V.: Privatising agricultural extension: Caveat emptor. In: Journal of Rural Studies 16/2000. S.95-102.

Knickel, K. / Peter, S.: Amenity-led development of rural areas: The example of the Regional Action pilot programme in Germany. In: Green, G. P. / Marcouiller, D. / Deller, S. (Hrsg.): Amenities and rural development: Theory, methods and public policy. Series: New Horizons in Environmental Economics. Northampton 2005. S.302-321.

Knickel, K. / Schiller, S. / Von Münchhausen, S. / Vihinen, H. / Weber A.: New institutional arrangements in rural development. In: van der Ploeg, J. D. / Marsden, T. (Hrsg.): Unfolding webs. Assen: Royal Van Gorcum 2008. S. $111-129$

Knickel, K. / Brunori, G. / Rand, S. / Proost, J.: Towards a better conceptual framework for innovation processes in agriculture and rural development: From linear models to systemic approaches. Journal of Agricultural Education and Extension. 2009 (im Druck).

Knickel, K. / Tisenkopfs, T. (Hrsg.): Creating innovation systems that support transitions towards a sustainable development: Acknowledging the embeddedness of innovation processes. Final project report (in Vorbereitung). Frankfurt 2009.

Oostindie, H. / Broekhuizen, R.: The dynamics of novelty production. In: van der Ploeg, J. D. / Marsden, T. (Hrsg.): Unfolding webs. Assen: Royal Van Gorcum 2008. S. 68-86

Rand, S. / Bourdin, D. / Brunori, G. / Dockès, A.-C. / Fischler, M. / Guillaumin, A. / Neri, T. / Sumane, S.: Environmental technologies. Synthesis report. IN-SIGHT project. Frankfurt am Main 2008.

van der Ploeg, J. D. / Renting, H. / Brunori, G. / Knickel, K. / Mannion, J. / Marsden, T. / De Roest, K. / Sevilla Guzman, E. / Ventura, F.: Rural development: From practices and policies towards theory. In: Sociologia Ruralis 40, 4/2000. S.391-408

van der Ploeg, J. D.: The virtual farmer: Past, present and future of the Dutch peasantry. Series: European Perspectives on Rural Development. Assen: Royal Van Gorcum 2003.

van der Ploeg, J. D. / Broekhuizen, R. / Brunori, G. / Sonnino, R. / Knickel, K. / Tisenkopfs, T. / Oostindie, H.: Towards a framework for understanding regional rural development. In: van der Ploeg, J. D. / Marsden, T. (Hrsg.): Unfolding webs. Assen: Royal Van Gorcum 2008. S. 1-28.

Wilson, G.: Multifunctional agriculture: A transition theory perspective. Wallingford 2007.

Wiskerke, H. / van der Ploeg, J. D.: Seeds of transition: Essays on novelty production, niches and regimes in agriculture. Assen 2004.

\section{AUTOREN + KONTAKT}

Dr. Karlheinz Knickel war, zuletzt als Geschäftsführer, am Institut für Ländliche Strukturforschung (IfLS) an der Universität Frankfurt am Main tätig, und arbeitet seit Dezember 2008 als Senior Policy Advisor im Sustainability Outcomes Team des neuseeländischen Umweltministeriums in Wellington.

Dr. Karlheinz Knickel, Ministry for the Environment, Sustainability Outcomes Team, Wellington, New Zealand, E-Mail: Karlheinz.Knickel@mfe.govt.nz

Dipl.-Soz. Sarah Peter ist Doktorandin an der
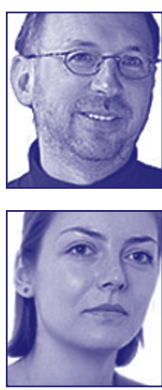
Universität Frankfurt am Main und ist im wissenschaftlichen Team des Instituts für Ländliche Strukturforschung (IfLS) tätig.

Sarah Peter, Institut für Ländliche Strukturforschung (IfLS) an der Johann Wolfgang Goethe Universität Frankfurt am Main, Zeppelinallee 31, 60325 Frankfurt am Main, Tel.: +49 69 775001, www.ifls.de, E-Mail: sarahp@stud.uni-frankfurt.de 
(c) 20I0 Authors; licensee IÖW and oekom verlag. This is an article distributed under the terms of the Creative Commons Attribution Non-Commercial No Derivates License (http://creativecommons.org/licenses/by-nc-nd/3.o/), which permits unrestricted use, distribution, and reproduction in any medium, provided the original work is properly cited. 